\title{
Persimmon Leaf Flavonols Enhance the Anti-Cancer Effect of Heavy Ion Radiotherapy on Murine Xenograft Tumors
}

\author{
Kayoko Kawakami $^{1}$, Hiroshi Nishida ${ }^{{ }^{*}}$, Naoto Tatewaki ${ }^{1}$, Kiyomi Eguchi-Kasai ${ }^{2}$, Kazunori Anzai ${ }^{2}$, \\ Takahiro Eitsuka ${ }^{1}$, Tetsuya Konishi ${ }^{1}$, Masao Hirayama ${ }^{1}$ \\ ${ }^{1}$ Laboratory of Nutritional Biochemistry, Niigata University of Pharmacy \& Applied Life Sciences, Niigata, Japan; ${ }^{2}$ National Insti- \\ tute of Radiological Sciences, Chiba, Japan. \\ Email: "wm7h-nsd@asahi-net.or.jp
}

Received June 27 $7^{\text {th }}$, 2013; revised July 28 ${ }^{\text {th }}$, 2013; accepted August $6^{\text {th }}, 2013$

Copyright $(2013$ Kayoko Kawakami et al. This is an open access article distributed under the Creative Commons Attribution License, which permits unrestricted use, distribution, and reproduction in any medium, provided the original work is properly cited.

\begin{abstract}
The cell cycle checkpoint system play a pivotal role in the cellular DNA damage response, and the discovery of checkpoint inhibitors is expected to sensitize current cancer therapies. Checkpoint signaling cascades are critically modulated by ATM (ataxia telangiectasia-mutated) and its related molecules. Generally, ATM primarily responds to ionizing irradiation-induced DNA double-strand breaks. Heavy ions from an accelerated carbon ion beam have been used to cure cancer because they are more effective than ionizing irradiation such as X-ray and $\gamma$-radiation in terms of biological damage. In a previous study, we demonstrated that a persimmon leaf flavonol (PLF) promoted the cytotoxic effect of chemotherapeutic agents on cancer cells through inhibition of checkpoint activities, especially in the ATM dependent pathway. The present study investigated whether PLF inhibits checkpoint activity during the DNA damage response induced by heavy ion irradiation. Treatment with PLF significantly increased the cytotoxicity of heavy ion irradiation in A549 adenocarcinoma cells. The phosphorylation of checkpoint proteins such as p53, SMC1, and Chk1 was increased by heavy ions. PLF reduced the phosphorylation of checkpoint proteins. Pre-treatment with PLF significantly prevented the decrease of mitotic cells in heavy ion-exposed cells. We further evaluated tumor volume in SCID mice inoculated with human lung adenocarcinoma A549 cells. The combination treatment of PLF and heavy ion resulted in a decrease of tumor volume compared with controls, although PLF itself did not exhibit any effect. These results indicate that PLF inhibits tumor growth through modulation of the DNA damage response. PLF may be useful for clinical application in combination with heavy ion radiotherapy.
\end{abstract}

Keywords: DNA Damage; Checkpoint; Persimmon Leaf Flavonols; Heavy Ion Irradiation; Xenograft

\section{Introduction}

Heavy ion radiotherapy has recently received increasing attention in the field of radiotherapy because heavy ion irradiation is more effective than other types of ionizing irradiation such as $\mathrm{X}$-ray or $\gamma$-radiation in inducing biological damage [1]. The carbon ion beam used in heavy ion irradiation releases a large amount of energy at the end of its range and enables the safe delivery of an effective dose of irradiation to cancer tissues without damaging normal tissues [2]. To date, more than 96,000 patients have been treated with particle beams around the world, of which approximately $10 \%$ were treated with

"Corresponding author. carbon ion radiotherapy [3].

It is already known that radiotherapy induces apoptosis by DNA damage [4]. At the DNA damage checkpoint, ATM (ataxia telangiectasia mutated) and ATR (AT and Rad3 related) play a central role in intracellular signal transduction and control the cell cycle precisely, by phosphorylating downstream components of the checkpoint pathway [5-7]. When DNA damage occurs, checkpoint effector proteins such as p53, structural maintenance of chromosomes 1 (SMC1), and checkpoint kinase 1 (Chk1) are phosphorylated and activated by ATM or ATR, which leads to cell cycle arrest in the G1, S, G2, and M phase, respectively. Thus, the DNA damage checkpoint induces cell cycle arrest and/or apoptosis. Furthermore, 
the G2/M checkpoint, which is usually preserved even in p53-defective or mutated cancer cells, plays an important role in the DNA damage checkpoint system [8]. Therefore, modulation of DNA damage checkpoint activity is an important target to improve the efficiency of cancer treatment.

We have focused on DNA damage checkpoint activity and have tried to discover natural compounds to sensitize cancer treatment [9]. In a previous study, we demonstrated that a flavonol contained in persimmon (Diospyros kaki) leaf extract (PLF) promoted the cytotoxic effect of chemotherapeutic agents on cancer cells by inhibiting checkpoint activity, especially in the ATM-dependent pathway [10]. Persimmon leaves are consumed as health promoting tea in Asia, and are reported to have various biological effects such as anti-oxidative [11-13], anticancer [14,15], anti-hypertensive [16,17], anti-inflammatory $[18,19]$ and anti-hyperglycemic [20,21]. PLF contains eight flavonols, and characterized by content of four 2"-galloylated flavonols [22]. Until now, there has been no practical source of plants readily available for the preparation of 2"-galloylated flavonols. PLF enables to further study of the biological activities of galloylated flavonols.

In this study, we investigated whether PLF inhibits checkpoint activity during the DNA damage response induced by heavy ion irradiation. Furthermore, we evaluated the inhibitory activity of PLF and heavy ion irradiation on the growth of A549 adenocarcinoma cells in a xenograft tumor model.

\section{Materials and Methods}

\subsection{Preparation of Persimmon Leaf Flavonols}

PLF was prepared as described previously [20]. Persimmon leaves were collected at Niitsu in Niigata, Japan. Dried persimmon leaves were treated with boiling water for $30 \mathrm{~min}$. The soluble extract was subsequently partitioned with ethyl acetate to give PLF. The ethyl acetate phase was evaporated to dryness in vacuo. The eight flavonol components in PLF consisted of four non-galloylated flavonols (kaempferol 3-O-glucoside, kaempferol 3-O-galactoside, quercetin 3-O-glucoside, and quercetin 3 -O-galactoside) and four galloylated flavonols (their 2"gallolyated flavonol glycosides). The eight flavonols in PLF were identified and quantified as described previously [22]. The percentage (\% w/w) of flavonols in PLF were $29 \%$ kaempferol 3-O-2"-galloylglucoside, 16\% kaempferol 3-O-2"-galloylgalactoside, $10 \%$ quercetin 3$O$-2"-galloylglucoside, $4 \%$ quercetin 3-O-2"-galloylgalactoside, $18 \%$ kaempferol 3-O-glucoside, $11 \%$ kaempferol 3-O-galactoside, $7 \%$ quercetin 3-O-glucoside, and $4 \%$ quercetin 3 - $O$-galactoside.

\subsection{Irradiation}

The carbon ion beam was generated by the Heavy Ion Medical Accelerator in Chiba (HIMAC) synchrotron at the National Institute of Radiological Sciences (NIRS), Japan. The $135 \mathrm{MeV} / \mathrm{u}$ heavy ion beams and $290 \mathrm{MeV} / \mathrm{u}$ beams with a width of $60 \mathrm{~mm}$ for the spread-out Braggpeak (SOBP) were used cell and animal experiments, respectively. The dosimetry and LET settings/measurements were determined by NIRS engineers.

\subsection{Cell Culture}

Human adenocarcinoma A549 cells were obtained from the American Type Culture Collection (ATCC; Manassas, VA, USA). The cells were cultured in Dulbecco's modified Eagle's medium (DMEM) (Sigma-Aldrich, MO, USA) containing $10 \%$ fetal bovine serum, $100 \mathrm{U} / \mathrm{mL}$ penicillin, and $100 \mu \mathrm{g} / \mathrm{mL}$ streptomycin at $37^{\circ} \mathrm{C}$ in a humidified atmosphere of $5 \% \mathrm{CO}_{2}$. The cells seeded in T25 culture flasks (Becton Dickinson, CA, USA) before irradiation.

\subsection{Clonogenic Assay}

Cell viability was determined by clonogenic assay. A549 cells were cultured for $24 \mathrm{~h}$ before a 1-h pretreatment with PLF $(0,10 \mu \mathrm{g} / \mathrm{mL})$ and subsequently exposed to heavy ion (0, 2.5, and 6 Gy). After irradiation, the cells were plated at 500 cells/60-mm dish. After incubation for 14 days, colonies were stained with $2 \%$ methylene blue in $50 \%$ ethanol. Clonogenic survival is expressed as a proportion of the colony number on control dishes.

\subsection{Immunoblot Analysis}

Intracellular levels of phosphorylation for each checkpoint proteins such as SMC1, Chk1, p53, and ATM were determined by western blotting using phospho-specific antibodies targeting Ser966, Ser317, Ser15, and Ser1981, respectively. A549 cells were cultured for $24 \mathrm{~h}$ before a 1-h pretreatment with PLF $(0,1,10$, and $30 \mu \mathrm{g} / \mathrm{mL})$ and subsequently exposed to heavy ion (0, 2.5, and $10 \mathrm{~Gy})$. After irradiation for $1 \mathrm{~h}$, cells were lysed in UTB buffer (8 mM urea, $150 \mathrm{mM}$ 2-mercaptorthanol, $50 \mathrm{mM}$ Tris$\mathrm{HCl}, \mathrm{pH}$ 7.5), and protein concentrations were determined using the Bradford protein assay kit (Bio-Rad Laboratories, CA, USA). For immunoblot analysis, the prepared proteins were separated by SDS polyacrylamide gel electrophoresis (SDS-PAGE) and transferred electrophoretically to a PVDF membrane. The membrane was blocked by $2 \%$ skim milk in Tris buffered saline containing $0.1 \%$ Tween 20 for $1 \mathrm{~h}$ at room temperature. Immunoblotting was performed using primary antibodies against phospho-SMC1 (Bethyl, TX, USA), phosphoChk1 (Bethyl), phospho-p53 (Cell Signaling Technology, 
MA, USA), phospho-ATM (Cell Signaling Technology), and GAPDH (Cell Signaling Technology) for $4 \mathrm{~h}$ at room temperature. Bound antibodies were detected using a secondary peroxidase-conjugated anti-rabbit or anti-mouse IgG (Cell Signaling Technology). Target proteins were visualized using an ECL reaction solution (Millipore Co., MA, USA) and a chemiluminescence-sensitive film (Fujifilm Co., Ltd., Tokyo, Japan).

\subsection{Flow Cytometric Analysis of the Cell Cycle}

The cells were treated with PLF for $24 \mathrm{~h}$ and then evaluated for DNA content after propidium iodide staining. The cell cycle distribution was not altered by treatment with PLF (data not shown).

\subsection{G2/M Checkpoint Analysis}

Phosphorylation of histone H3 at Ser10 was used to monitor mitosis. A549 cells were incubated with PLF ( 0 and $30 \mu \mathrm{g} / \mathrm{mL})$ at $1 \mathrm{~h}$ prior to heavy ion treatment $(0,2.5$, and 10 Gy). After treatment, cells were suspended in $300 \mu \mathrm{L}$ of PBS and $700 \mu \mathrm{L}$ of ice-cold ethanol and permeabilized with $0.25 \%$ Triton X-100 in PBS on ice for 15 min. The cells were then incubated with polyclonal anti-rabbit phospho-histone H3 (Ser10) antibody (Upstate, NY, USA) and Alexa Fluor 488-conjugated goat anti-rabbit IgG antibody (Invtrogen, CA, USA) for $4 \mathrm{~h}$ and $1 \mathrm{~h}$ at room temperature, respectively. The cellular DNA was stained by $50 \mu \mathrm{g} / \mathrm{mL}$ propidium iodide (Dojindo, Kumamoto, Japan) for $30 \mathrm{~min}$ at room temperature. The stained cells were analyzed by flow cytometer (Beckman-Coulter, CA, USA).

\subsection{Xenograft Study}

Five-week-old male SCID mice (C.B-17/lcr-scid/ scidJcl) were purchased from CLEA Japan (Tokyo, Japan). The animals were housed at $24^{\circ} \mathrm{C} \pm 1^{\circ} \mathrm{C}$ with a $12 \mathrm{~h}$ light:dark cycle and had free access to a standard diet and distilled water for 1 week prior to the experiment. Animal experimental protocols were approved by the NIRS Institutional Animal Care and Use Committee, and the study was conducted in accordance with the Animal Experimentation Guidelines of NIRS.

A549 cells were subcutaneously injected $\left(5 \times 10^{6}\right.$ cells per mouse) into the left hind leg of mice, and tumor size was measured using a Vernier caliper. Treatments were initiated when tumors were established $\left(\sim 150 \mathrm{~mm}^{3}\right)$ by 21 days after tumor implantation, and the mice were randomized to control and treatment groups. Nonanesthetized mice were immobilized on a lucite plate to place their right hind legs in the irradiation field and then exposed to heavy ions (2.5 Gy). The PLF treatment group was orally administered PLF (100 mg/kg body weight) using a stomach tube every day for total of 10 days. The body weights and tumor sizes were recorded every day.

\subsection{Statistics}

The data were expressed as the mean \pm SE. Statistical analysis of the data was performed by ANOVA followed by the Tukey test to identify differences among groups. Differences were considered significant at $P<0.05$.

\section{Results}

\subsection{Effect of PLF Treatment on Cell Viability after Heavy Ion Irradiation}

We investigated whether PLF affects cell viability after heavy ion irradiation. The viability of A549 cells was measured by clonogenic assay. Heavy ion irradiation induced cell death in a dose dependent manner (Figure 1). When the cells were treated with $10 \mu \mathrm{g} / \mathrm{mL}$ PLF before 6 Gy irradiation, their viability significantly decreased relative to untreated control cells. PLF-only treatment did not affect viability and cell cycle progression (data not shown). These results indicate that PLF sensitizes the cytotoxicity induced by heavy ion irradiation.

\subsection{Effect of PLF on the Phosphorylation of Checkpoint Proteins after Heavy Ion Irradiation}

We examined the effect of PLF on DNA damage check point pathways induced by heavy ion irradiation. Heavy ion irradiation increased the phosphorylation of the checkpoint proteins such as SMC1, Chk1, and p53 at Ser966, Ser317, and Ser15, respectively (Figure 2, lane 2). When the cells were exposed to 2.5-Gy heavy ion radiation, PLF treated cells dose-dependently decreased phosphorylation of SMC1, Chk1, and p53 (Figure 2(a)).

We further tested the effect of PLF on the phosphorylation of ATM (Ser1981), a key molecule in DNA damage checkpoint signaling. Heavy ion irradiation slightly increased phosphorylation of ATM at Ser1981 (Figure 2(a), lane 2). PLF dose-dependently decreased ATM phosphorylation (Figure 2(a), line 4).

PLF also decreased the phosphorylation of p53 after 10 Gy of heavy ion (Figure 2(b), line 3), although it did not affect the phosphorylation of SMC1 (Figure 2(b), line 1). PLF at $30 \mu \mathrm{g} / \mathrm{mL}$ decreased Chk1 phosphorylation though 1 and $10 \mu \mathrm{g} / \mathrm{mL}$ slightly increased Chk1 phosphorylation (Figure 2(b), line 2).

\subsection{Effect of PLF on the G2/M Checkpoint after Heavy Ion Irradiation}

To evaluate the specificity of the inhibitory effect of PLF on the cellular DNA damage response, we further examined the mitotic transition using flow cytometric analysis (Figure 3). The percentage of mitotic cells was estimated 


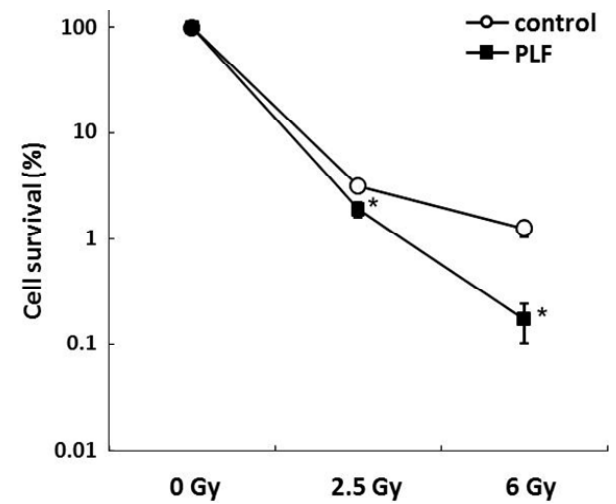

Figure 1. PLF treatment decreases cell viability after DNA damage. Relative cell viability was measured by a clonogenic assay. A549 cells were subjected to $1 \mathrm{~h}$ of pre-incubation and 14 days of post-incubation with PLF $(10 \mu \mathrm{g} / \mathrm{mL})$ after heavy ion (2.5 and $6 \mathrm{~Gy})$. The values given are the mean \pm SE $(n=4) .{ }^{*} P<0.05$ vs. PLF untreated control.

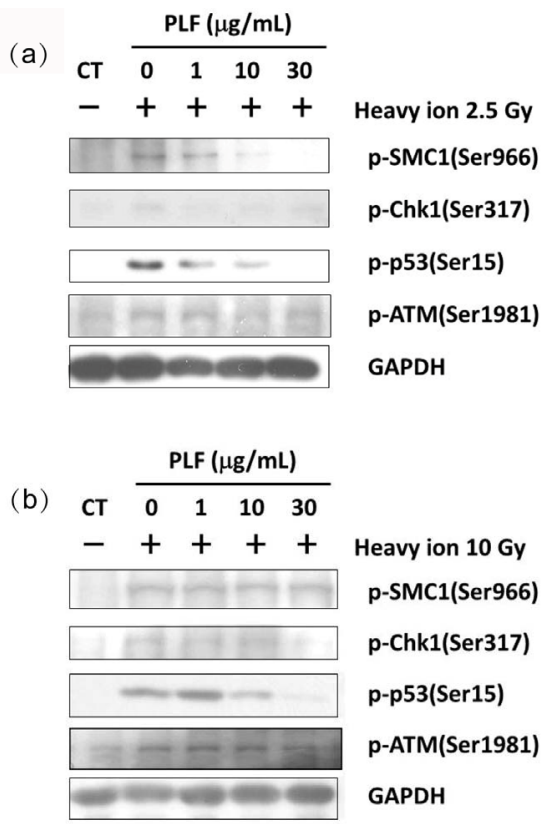

Figure 2. Inhibitory effects of PLF treatment on checkpoint proteins after heavy ion irradiation. The phosphorylation of SMC1 (Ser966), Chk1 (Ser317), p53 (Ser15), and ATM (Ser1981) was determined by immunoblotting analysis. Dose-dependent effects of PLF on the phosphorylation of SMC1, Chk1, p53, and ATM were observed by adding increasing concentrations of PLF before and after induction of DNA damage by heavy ion irradiation ((a) $2.5 \mathrm{~Gy}$ and (b) 10 Gy).

according to the level of histone $\mathrm{H} 3$ phosphorylation at Ser10. The percentage of phospho-histone H3-positive cells clearly decreased after heavy ion irradiation (Figure 3(a)). In contrast, the percentage of PLF-treated cells undergoing mitosis significantly increased 2.5-Gy irradiation. The percentage of mitotic cells in PLF-pretreated cells increased compared to the $10 \mathrm{~Gy}$ of irradiation-only

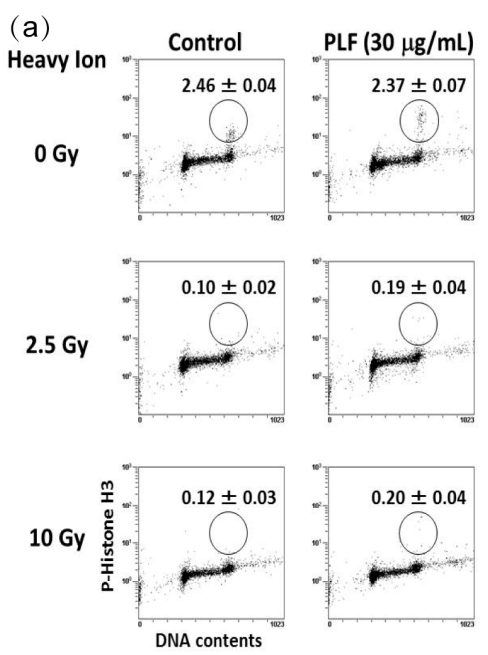

(b)

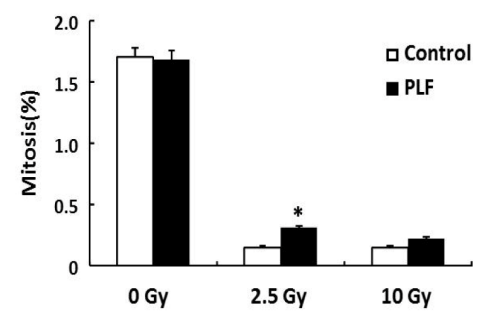

Figure 3. Effect of PLF on G2/M checkpoint activity. (a) Dot plot analysis was performed by FACS followed by data analysis. The percentage of mitotic cells was estimated by phosphorylated histone H3 (Ser10) positive cells. The cells were counter stained with PI for DNA content. A549 cells were treated with $30 \mu \mathrm{g} / \mathrm{mL}$ PLF for $1 \mathrm{~h}$ before and $1 \mathrm{~h}$ after heavy ion irradiation (0, 2.5, and $10 \mathrm{~Gy})$. (b) Data are expressed as the percentage of mitotic cells with respect to the total number of cells. The values given are the mean \pm SE $(n=4) .{ }^{*} P<0.01$ vs. PLF untreated control.

cells, but there was no statistically significant difference (Figure 3(b)). These results suggest that PLF abrogated the G2/M checkpoint in heavy ion irradiation induced DNA damage.

\subsection{Effect of PLF on Tumor Growth after Heavy Ion Irradiation}

We next assessed the effect of oral administration of PLF on tumor growth in heavy ion exposure using a murine xenograft model. A marked change in body weight was not observed between the groups (data not shown). The tumor volume of the control group reached $249 \pm 26$ $\mathrm{mm}^{3}$ by day 31 after tumor transplantation (Figure 4). Tumor growth was inhibited in the heavy ion irradiation group after day 25. The administration of PLF alone did not affect tumor volume. In contrast, combined PLF and heavy ion treatment significantly reduced the tumor volume compared to the control. The final mean tumor volume for PLF and heavy ion-treated mice was $155 \pm$ $19 \mathrm{~mm}^{3}$, which was $38 \%$ less than the control group with 


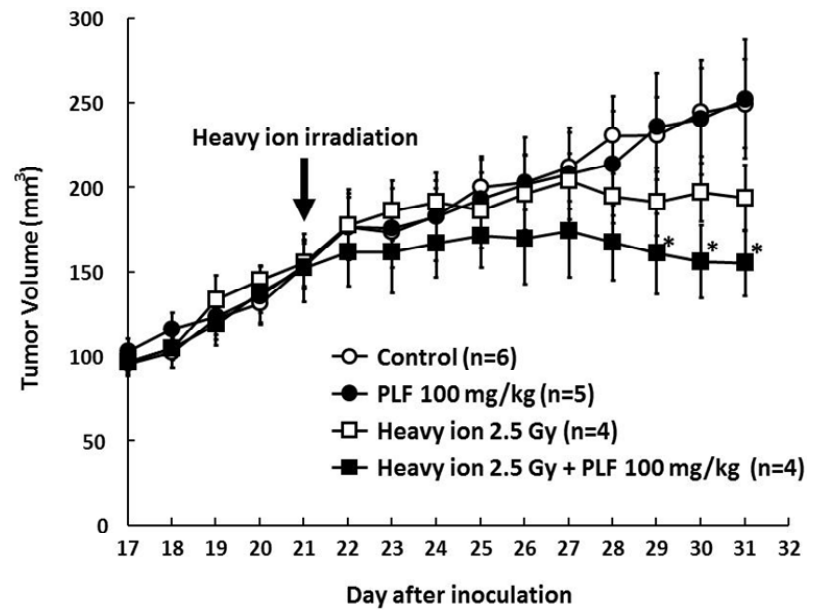

Figure 4. Tumor growth curve of mice treated with PLF, heavy ion irradiation, or a combination of PLF and irradiation. Tumor-bearing SCID mice were randomly assigned to the indicated treatment groups (PLF and irradiation untreated control, PLF treatment, irradiation, and combined PLF and irradiation treatment). The 2.5-Gy heavy ion irradiation was performed on day 21 as indicated by the arrow, and tumor volume was measured. The PLF-treated group was administrated $100 \mathrm{mg} / \mathrm{kg}$ PLF every day after irradiation. The values given are the means \pm SE $(n=4-6) .{ }^{*} P<$ 0.05 vs. untreated control.

a mean of $249 \pm 26 \mathrm{~mm}^{3}$. These results suggest that PLF might be applicable to the sensitization of radiotherapy.

\section{Discussion}

In a previous study, we reported that PLF promoted the cytotoxic effect of chemotherapeutic agents doxorubicin (DOX) on cancer cells by inhibiting checkpoint activity [10]. Moreover, we demonstrated that PLF inhibited ATM-dependent checkpoint activity in DOX-induced DNA damage responses. In checkpoint signaling, ATM plays a key role and critically modulates many events in the cell cycle through the phosphorylation of effector proteins [5,6]. Consequently, the modulation of DNA damage checkpoint activity in cancer cells is an important target for sensitization of cancer therapy. Here, we investigated whether PLF inhibits checkpoint activity during the DNA damage response induced by heavy ion irradiation.

We demonstrated that heavy ion irradiation-induced cell death was significantly sensitized by PLF treatment (Figure 1). Heavy ion irradiation induced DNA doublestrand break, and ATM was activated [23-25]. In this study, we investigated whether 2.5-Gy and 10-Gy heavy ion irradiation affected the phosphorylation of ATM and its downstream checkpoint proteins such as SMC1, Chk1, and p53. In particular, 2.5-Gy heavy ion irradiation induced the phosphorylation of ATM and the downstream proteins (Figure 2(a)). These results indicate that ATM plays an important role in the DNA damage response induced by heavy ion radiation. Furthermore, PLF treatment decreased phosphorylation of each molecule. In contrast, the effects were not obvious in high-dose (10 Gy) irradiation (Figure 2(b)). Therefore, ATM may not be responsible for the subsequent cellular DNA damage response to high-intensity heavy ion irradiation, even though the damage is severe enough to induce checkpoint activation. It is well known that ATM is not the only molecule involved in the response to cellular DNA damage; ATR, which plays a central role in intracellular signal transduction, also plays a role [5-7]. We previously showed that PLF did not have specific effect on ATR activity [10]. Hence, it is likely that other molecules, such as ATR, play a key role in the DNA damage response induced by high-dose (more than $10 \mathrm{~Gy}$ ) irradiation. In addition, it has been reported that clustered DNA damage greater than simple double-strand breaks is likely to occur at biologically relevant frequencies in response to irradiation [26,27]. Because high-dose irradiation (10 Gy) occasionally exerts a complicated activation of the DNA damage response, PLF or other compounds that specifically modulate a single molecule might not produce a significant effect on overall signal transduction.

It has also been established that the mutation or deletion of p53 and the lack of the G1/S checkpoint frequently occurs in cancer cells [28]. Therefore, the G2/M checkpoint is considered to be a pivotal target for cancer therapies [29-31]. The present study demonstrated that PLF abrogates the G2/M checkpoint of the DNA damage response to low-dose (2.5 Gy) irradiation through inhibition of ATM. This finding suggests that PLF is useful to increase the cytotoxicity of heavy ion irradiation in tumor cells that do not have functional p53 as a DNA damage checkpoint molecule.

We further compared the growth rate of xenograft made by transplantation of A549 cells. Because PLF modulates the activity of DNA damage checkpoints activated by low-dose irradiation, we used 2.5-Gy heavy ion irradiation. Interestingly, oral administration of PLF itself did not seem to have a therapeutic effect, however, the combination of PLF and heavy ion treatment resulted in significant tumor regression (Figure 4). Taken together, these results suggest that the antitumor effect observed with combined PLF and irradiation was caused by the abrogation of DNA damage checkpoints. Therefore, a combination treatment produced more effective outcomes than heavy ion irradiation treatment alone. PLF contains four 2"-galloylat1ed flavonols, which have been reported to have interesting biological activities, including xanthine oxidase inhibitory activity [32], antioxidant activity [22], HIV-1 integrase inhibitory activity [33], and inhibitory activity against inducible nitric oxide synthase expression [34]. Thus far, 2"-galloylated flavonols have 
been isolated from Terminalia chebula [33] and Persicaria lapathifolia [34], but not from edible plant. Because persimmon leaf tea is popularly consumed as beverage, we can easily take 2"-galloylated flavonols. However, to understand the bioavailability of 2"-galloylated flavonols, further study will be required.

In conclusion, we demonstrated that PLF inhibit tumor growth by modulation of DNA damage checkpoint in vitro and in vivo. Several studies have reported that chemical agents sensitize radiotherapy by abrogation of DNA damage checkpoint [35-38]. Almost $60 \%$ of drugs approved for cancer treatment are derived from natural products or natural product analogues [39]. It is expected that natural products have a lower risk of side effects than chemical agents. 2"-Galloylated flavonols are natural products from persimmon leaf, which is commonly consumed as tea in Asia. PLF administration has a lower risk of side effects than administration of other anticancer agents. The combination with PLF and heavy ion irradiation may permit a reduction of the doses of irradiation administered to cancer patients, thereby decreasing the severity of side effects.

\section{Acknowledgements}

This work was supported by the Heavy Ions research project at NIRS-HIMAC. We thank Professor Osamu Inanami and Dr. Hironobu Yasui, Hokkaido University for kind assistance with the xenograft study. This work was partly supported by a Grant-in-Aid for Scientific research (C) (KAKENHI \#24580203) and the Strategic Research Foundation at Private Universities (\#S1001030) from the Ministry of Education, Culture, Sports, Science, and Technology (MEXT) of Japan.

\section{REFERENCES}

[1] P. Todd, J. C. Wood, J. T. Walker and S. J. Weiss, "Lethal, Potentially Lethal, and Nonlethal Damage Induction by Heavy Ions in Cultured Human Cells," Radiation Research Supplement, Vol. 8, 1985, pp. S5-S12. doi: $10.2307 / 3576625$

[2] N. Hamada, T. Imaoka, S. Masunaga, T. Ogata, R. Okayasu, A. Takahashi, T. A. Kato, Y. Kobayashi, T. Ohnishi, K. Ono, Y. Shimada and T. Teshima, "Recent Advances in the Biology of Heavy-Ion Cancer Therapy," Journal of Radiation Research, Vol. 51, No. 4, 2010, pp. 365-383. doi:10.1269/jrr.09137

[3] T. Ohno, "Particle Radiotherapy with Carbon Ion Beams," EPMA Journal, Vol. 4, No. 1, 2013, p. 9. doi:10.1186/1878-5085-4-9

[4] G. M. Ross, "Induction of Cell Death by Radiotherapy," Endocrine-Related Cancer, Vol. 6, No. 1, 1999, pp. 4144. doi:10.1677/erc.0.0060041

[5] Y. Shiloh, "ATM and ATR: Networking Cellular Responses to DNA Damage," Current Opinion in Genetics
\& Development, Vol. 11, No. 1, 2001, pp. 71-77. doi:10.1016/S0959-437X(00)00159-3

[6] R. T. Abraham, "Cell Cycle Checkpoint Signaling through the ATM and ATR Kinases," Genes \& Development, Vol. 15, No. 17, 2001, pp. 2177-2196. doi:10.1101/gad.914401

[7] S. Matsuoka, B. A. Ballif, A. Smogorzewska, E. R. McDonald, K. E. Hurov, J. Luo, C. E. Bakalarski, Z. Zhao, N. Solimini, Y. Lerenthal, Y. Shiloh, S. P. Gygi and S. J. Elledge, "ATM and ATR Substrate Analysis Reveals Extensive Protein Networks Responsive to DNA Damage," Science, Vol. 316, No. 5828, 2007, pp. 1160-1166. doi:10.1126/science.1140321

[8] S. N. Powell, J. S. DeFrank, P. Connell, M. Eogan, F. Preffer, D. Dombkowski, W. Tang and S. Friend, "Differential Sensitivity of p53(-) and p53(+) Cells to Caffeine-Induced Radiosensitization and Override of G2 Delay,” Cancer Research, Vol. 55, No. 8, 1995, pp. 16431648.

[9] H. Nishida, N. Tatewaki, Y. Nakajima, T. Magara, K. M. Ko, Y. Hamamori and T. Konishi, "Inhibition of ATR Protein Kinase Activity by Schisandrin B in DNA Damage Response,” Nucleic Acids Research, Vol. 37, No. 17, 2009, pp. 5678-5689. doi:10.1093/nar/gkp593

[10] K. Kawakami, H. Nishida, N. Tatewaki, Y. Nakajima, T. Konishi and M. Hirayama, "Persimmon Leaf Extract Inhibits the ATM Activity during DNA Damage Response Induced by Doxorubicin in A549 Lung Adenocarcinoma Cells," Bioscience, Biotechnology, and Biochemistry, Vol. 75, No. 4, 2011, pp. 650-655. doi:10.1271/bbb.100738

[11] S. Sakanaka, Y. Tachibana and Y. Okada, "Preparation and Antioxidant Properties of Extracts of Japanese Persimmon Leaf Tea (Kakinoha-Cha)," Food Chemistry, Vol. 89, No. 4, 2005, pp. 569-575. doi:10.1016/j.foodchem.2004.03.013

[12] W. Bei, W. Peng, Y. Ma and A. Xu, "Flavonoids from the Leaves of Diospyros kaki Reduce Hydrogen PeroxideInduced Injury of NG108-15 Cells,” Life Science, Vol. 76, No. 17, 2005, pp. 1975-1988. doi:10.1016/j.lfs.2004.09.031

[13] L. Sun, J. Zhang, X. Lu, L. Zhang and Y. Zhang, "Evaluation to the Antioxidant Activity of Total Flavonoids Extract from Persimmon (Diospyros kaki L.) Leaves,” Food and Chemical Toxicology, Vol. 49, No. 10, 2011, pp. 2689-2696. doi:10.1016/j.fct.2011.07.042

[14] G. Chen, J. Xue, S. X. Xu and R. Q. Zhang, "Chemical Constituents of the Leaves of Diospyros kaki and Their Cytotoxic Effects," Journal of Asian Natural Products Research, Vol. 9, No. 3-5, 2007, pp. 347-353. doi:10.1080/10286020600727731

[15] P. Khanal, W. K. Oh, P. T. Thuong, S. D. Cho and H. S. Choi, "24-Hydroxyursolic Acid from the Leaves of the Diospyros kaki (Persimmon) Induces Apoptosis by Activation of AMP-Activated Protein Kinase,” Planta Medica, Vol. 76, No. 7, 2010, pp. 689-693. doi:10.1055/s-0029-1240678

[16] K. Kameda, T. Takaku, H. Okuda, Y. Kimura, T. Okuda, T. Hatano, I. Agata and S. Arichi, "Inhibitory Effects of Various Flavonoids Isolated from Leaves of Persimmon 
on Angiotensin-Converting Enzyme Activity,” Journal of Natural Products, Vol. 50, No. 4, 1987, pp. 680-683. doi:10.1021/np50052a017

[17] K. Kawakami, S. Aketa, H. Sakai, Y. Watanabe, H. Nishida and M. Hirayama, "Antihypertensive and Vasorelaxant Effects of Water-Soluble Proanthocyanidins from Persimmon Leaf Tea in Spontaneously Hypertensive Rats," Bioscience, Biotechnology, and Biochemistry, Vol. 75, No. 8, 2011, pp. 1435-1439. doi:10.1271/bbb.100926

[18] M. Kotani, M. Matsumoto, A. Fujita, S. Higa, W. Wang, M. Suemura, T. Kishimoto and T. Tanaka, "Persimmon Leaf Extract and Astragalin Inhibit Development of Dermatitis and IgE Elevation in NC/Nga Mice," Journal of Allergy and Clinical Immunology, Vol. 106, No. 1, 2000, pp. 159-166. doi:10.1067/mai.2000.107194

[19] M. Matsumoto, M. Kotani, A. Fujita, S. Higa, T. Kishimoto, M. Suemura and T. Tanaka, "Oral Administration of Persimmon Leaf Extract Ameliorates Skin Symptoms and Transepidermal Water Loss in Atopic Dermatitis Model Mice, NC/Nga," British Journal of Dermatology, Vol. 146, No. 2, 2002, pp. 221-227. doi:10.1046/j.1365-2133.2002.04557.x

[20] K. Kawakami, S. Aketa, M. Nakanami, S. Iizuka and M. Hirayama, "Major Water-Soluble Polyphenols, Proanthocyanidins, in Leaves of Persimmon (Diospyros kaki) and Their Alpha-Amylase Inhibitory Activity,” Bioscience, Biotechnology, and Biochemistry, Vol. 74, No. 7, 2010, pp. 1380-1385. doi:10.1271/bbb.100056

[21] U. J. Jung, Y. B. Park, S. R. Kim and M. S. Choi, "Supplementation of Persimmon Leaf Ameliorates Hyperglycemia, Dyslipidemia and Hepatic Fat Accumulation in Type 2 Diabetic Mice,” PLoS One, Vol. 7, No. 11, 2012, Article ID: e49030. doi:10.1371/journal.pone.0049030

[22] K. Kawakami, Y. Shibukura, T. Kanno, T. Furuki, S. Aketa and M. Hirayama, "Identification of 2"-Galloylated Flavonol 3-O-Glycosides Accumulating in Developing Leaves of Persimmon,” Phytochemical Analysis, Vol. 22, No. 5, 2011, pp. 403-410. doi:10.1002/pca.1295

[23] S. Kitajima, H. Nakamura, M. Adachi, K. Ijichi, Y. Yasui, N. Saito, M. Suzuki, K. Kurita and K. Ishizaki, "AT Cells Show Dissimilar Hypersensitivity to Heavy-Ion and XRays Irradiation,” Journal of Radiation Research, Vol. 51, No. 3, 2010, pp. 251-255. doi:10.1269/jrr.09069

[24] S. Ghosh, H. Narang, A. Sarma and M. Krishna, "DNA Damage Response Signaling in Lung Adenocarcinoma A549 Cells Following Gamma and Carbon Beam Irradiation," Mutation Research, Vol. 716, No. 1-2, 2011, pp. 10-19. doi:10.1016/j.mrfmmm.2011.07.015

[25] L, Xue, D. Yu, Y. Furusawa, J. Cao, R. Okayasu and S. Fan, "ATM-Dependent Hyper-Radiosensitivity in Mammalian Cells Irradiated by Heavy Ions,” International Journal of Radiation Oncology, Biology, Physics, Vol. 75, No. 1, 2009, pp. 235-243.

[26] D. T. Goodhead, "Initial Events in the Cellular Effects of Ionizing Radiations: Clustered Damage in DNA,” International Journal of Radiation Biology, Vol. 65, No. 1, 1994, pp. 7-17. doi:10.1080/09553009414550021

[27] M. Hada and A. G. Georgakilas, "Formation of Clustered DNA Damage after High-LET Irradiation: A Review,”
Journal of Radiation Research, Vol. 49, No. 3, 2008, pp 203-210. doi:10.1269/jrr.07123

[28] A. J. Levine, "p53, the Cellular Gatekeeper for Growth and Division," Cell, Vol. 88, No. 3, 1997, pp. 323-331. doi:10.1016/S0092-8674(00)81871-1

[29] Y. Wang, J. Li, R. N. Booher, A. Kraker, T. Lawrence, W. R. Leopold and Y. Sun, "Radiosensitization of p53 Mutant Cells by PD0166285, a Novel G(2) Checkpoint Abrogator," Cancer Research, Vol. 61, No. 22, 2001, pp. 8211-8217.

[30] T. Kawabe, "G2 Checkpoint Abrogators as Anticancer Drugs,” Molecular Cancer Therapeutics, Vol. 3, No. 4, 2004, pp. 513-519.

[31] D. P. Liu, H. Song and Y. Xu, “A Common Gain of Function of p53 Cancer Mutants in Inducing Genetic Instability,” Oncogene, Vol. 29, No. 7, 2010, pp. 949-956. doi:10.1038/onc.2009.376

[32] T. Hatano, T. Yasuhara, R. Yoshihara, Ikegami, Y. M. Matsuda, K. Yazaki, I. Agata, S. Nishibe, T. Noro and M. Yoshizaki, "Inhibitory Effects of Galloylated Flavonoids on Xanthine Oxidase,” Planta Medica, Vol. 57, No. 1, 1991, pp. 83-84. doi:10.1055/s-2006-960028

[33] M. J. Ahn, C. Y. Kim, J. S. Lee, T. G. Kim, S. H. Kim, C. K. Lee, B. B. Lee, C. G. Shin, H. Huh and J. Kim, "Inhibition of HIV-1 Integrase by Galloyl Glucoses from Terminalia Chebula and Flavonol Glycoside Gallates from Euphorbia pekinensis," Planta Medica, Vol. 68, No. 5, 2002, pp. 457-459. doi:10.1055/s-2002-32070

[34] H. Y. Jo, Y. Kim, S. Y. Nam, Lee, B. J. Y. B. Kim, Y. W. Yun and B. Ahn, "The Inhibitory Effect of Quercitrin Gallate on iNOS Expression Induced by Lipopolysaccharide in Balb/c Mice," Journal of Veterinary Science, Vol. 9, No. 3, 2008, pp. 267-272. doi:10.4142/jvs.2008.9.3.267

[35] A. J. Chalmers, E. M. Ruff, C. Martindale, N. Lovegrove and S. C. Short, "Cytotoxic Effects of Temozolomide and Radiation Are Additive- and Schedule-Dependent,” International Journal of Radiation Oncology, Biology, Physics, Vol. 75, No. 5, 2009, pp. 1511-1519. doi:10.1016/j.ijrobp.2009.07.1703

[36] V. Gavrilov, Y. Leibovich, S. Ariad, K. Lavrenkov and S. Shany, "A Combined Pretreatment of 1,25-Dihydroxyvitamin D3 and Sodium Valproate Enhances the Damaging Effect of Ionizing Radiation on Prostate Cancer Cells," Journal of Steroid Biochemistry and Molecular Biology, Vol. 121, No. 1-2, 2010, pp. 391-394. doi:10.1016/j.jsbmb.2010.03.004

[37] M. A. Morgan, L. A. Parsels, L. Zhao, Parsels, J. D. M. A. Davis, M. C. Hassan, S. Arumugarajah, L. HylanderGans, D. Morosini, D. M. Simeone, C. E. Canman, D. P. Normolle, S. D. Zabludoff, J. Maybaum and T. S. Lawrence, "Mechanism of Radiosensitization by the Chk1/2 Inhibitor AZD7762 Involves Abrogation of the G2 Checkpoint and Inhibition of Homologous Recombinational DNA Repair,” Cancer Research, Vol. 70, No. 12, 2010, pp. 4972-4981. doi:10.1158/0008-5472.CAN-09-3573

[38] S. E. Golding, E. Rosenberg, N. Valerie, I. Hussaini, M. Frigerio, X. F. Cockcroft, W. Y. Chong, M. Hummersone, L. Rigoreau, K. A. Menear, M. J. O’Connor, L. F. Povirk, T. van Meter and K. Valerie, "Improved ATM Kinase In- 
hibitor KU-60019 Radiosensitizes Glioma Cells, Compromises Insulin, AKT and ERK Prosurvival Signaling, and Inhibits Migration and Invasion,” Molecular Cancer Therapeutics, Vol. 8, No. 10, 2009, pp. 2894-2902.

doi:10.1158/1535-7163.MCT-09-0519
[39] D. J. Newman and G. M. Cragg, "Natural Products as Sources of New Drugs over the Last 25 Years,” Journal of Natural Products, Vol. 70, No. 3, 2007, pp. 461-477. doi:10.1021/np068054v 